\title{
GLOBAL WORLD FUNCTIONS
}

\author{
FRANCO CARDIN and ANTONIO MARIGONDA
}

\author{
Communicated by Mauro Spera
}

\begin{abstract}
Starting from the Amann-Conley-Zehnder finite reduction framework in the non-compact Viterbo's version we discuss the existence of global generating function with a finite number of auxiliary parameters describing the two-points Characteristic Relation related to the geodesic problem in the Hamiltonian formalism. This applies both to Analytical Mechanics and to General Relativity - we construct a global object generalizing the World Function introduced by Synge, which is well-defined only locally. Whenever the auxiliary parameters can be fully removed, Synge's World Function is restored.
\end{abstract}

\section{Introduction}

In the textbook by J. Synge [19] one can find the following definition:

Let $P^{\prime}\left(x^{\prime}\right)$ and $P(x)$ be two points in the space-time, joined by a geodesic $\Gamma$ with equations $x^{i}=\xi^{i}(u)$ where $u$ is a special ${ }^{1}$ parameter. Then the integral

$$
\Omega\left(P^{\prime} P\right)=\Omega\left(x^{\prime}, x\right)=\frac{1}{2}\left(u_{1}-u_{0}\right) \int_{u_{0}}^{u_{1}} g_{i j} U^{i} U^{j} \mathrm{~d} u
$$

taken along $\Gamma$ with $U^{i}=\mathrm{d} \xi^{i} / \mathrm{d} u$, has a value independent of the particular special parameter chosen. If, as we shall suppose, the points $P^{\prime}$ and $P$ determine a unique geodesic passing through them, then $\Omega$ is a function of these two points. As a function of the eight variables $x^{\prime}$ and $x$ we shall call it the world-function of space-time.

The World Function had a rather troubled history. The main criticism is that it has only a local meaning, and even in simple cases we cannot use it for global analysis. Really, Synge recognized this limitation a few lines after its definition.

\footnotetext{
${ }^{1}$ special parameters are the representative elements of a class of parameters invariant by affine transformations.
} 
He used this object for approximations and to solve geodesic triangles in spaces of small curvature. In more recent times, in the textbook by De Felice-Clarke [14], it is used in measure theory to define spatial length between an observer and an event, to measure the effect of curvature in the measure of angles, and in the study of the Doppler effect.

From a strictly analytical mechanical point of view, the World Function appears as the generating function of the canonical transformation induced by the differential equations of the geodesic system, thought of in their Hamiltonian format, and it exists whenever the phenomenon of the entanglement of geodesics in the base manifold (the space-time) does not occur. Such a generating function is solution of a certain Hamilton-Jacobi equation, and it has been a rather intriguing matter along past years to establish a useful notion of 'global solution' in HamiltonJacobi theory in a rigorous way. In order to give a comprehensible explanation of our proposal about globalization of the World Function, we briefly review a few elements of the geometric setting for the global theory of the Hamilton-Jacobi equation.

Given a Hamilton-Jacobi problem $H \circ \mathrm{d} S=e, H: T^{*} Q \rightarrow \mathbb{R}$, the symplectic geometry environment provides a new concept of solutions, called geometric solutions, i.e. Lagrangian submanifolds $\Lambda$ in the coisotropic fiber $H^{-1}(e)$ of $T^{*} Q$. These geometric solutions have not only local meaning. The description of the Lagrangian submanifolds is a crucial problem in the symplectic arena: a geometric solution of a Hamilton-Jacobi problem $H=e$ is meaningful - and then comparable to the classical (weak) solutions [4] - if we are able to write for it some generating functions, see e.g. [5-10,20]. A fundamental tool in this area is the Maslov-Hörmander theorem: it shows that we can locally describe Lagrangian submanifolds $\Lambda \subset T^{*} Q$ as image of the differential - with respect to $q \in Q$ of a smooth function $S=S(q, u)$, valued at the stationary points $u$ of a set of auxiliary parameters $u \in \mathbb{R}^{k}$, for a suitable integer $k \in \mathbb{N}$. In other words, locally at each point $\lambda \in \Lambda \subset T^{*} Q$

$$
\Lambda=\left\{\left(q^{i}, p_{j}\right) ; q^{i} \in Q, p_{j}=\frac{\partial S}{\partial q^{j}}\left(q^{i}, u^{A}\right), \text { for } u^{A} \text { s.t. } \frac{\partial S}{\partial u^{B}}\left(q^{i}, u^{A}\right)=0\right\}
$$

with the transversality condition

$$
\left.\operatorname{rk}\left(\begin{array}{ll}
\frac{\partial^{2} S}{\partial u^{A} \partial q^{i}} & \frac{\partial^{2} S}{\partial u^{A} \partial u^{B}}
\end{array}\right)\right|_{\Lambda}=k=\max .
$$

The importance of having global generating function $S$ is evident when we try to construct classical solutions of the Hamilton-Jacobi problem from geometric 
ones. In fact, if the relation

$$
\frac{\partial S}{\partial u^{B}}\left(q^{i}, u^{A}\right)=0
$$

can be solved with respect to $u^{A}$, i.e. if for every $q$ there exists one and only one $u^{A}=\tilde{u}^{A}(q)$ solving it, then we may define a classical (smooth) solution $\tilde{S}(q):=S(q, \tilde{u}(q))$. It is well known that in general such a classical solution does not exist globally (i.e. one cannot remove all auxiliary parameters $u$ ). This is due mainly to the nonlinearity of the problem (Hamiltonian function and initial data) and the related lack of transversality of $\Lambda$ with respect to the fibers of $\pi_{Q}$ : $T^{*} Q \rightarrow Q$. Nevertheless, when the above stationarization procedure of producing classical solutions fails, by the global generating function for $\Lambda$ one can try to build up viscosity solutions (in the sense of Crandall-Evans-Lions) by removing the auxiliary parameters through suitable inf-sup procedures, e.g.

$$
s=s(q):=\inf _{u^{A} \in \mathbb{R}^{k_{1}}} \sup _{u^{\alpha} \in \mathbb{R}^{k_{2}}} S\left(q, u^{A}, u^{\alpha}\right), \quad k_{1}+k_{2}=k .
$$

This program actually works in some interesting cases, see e.g. [4] and [9].

A first rigorous attempt to globalize the Maslov-Hörmander theorem was made by Laudenbach [16], Sikorav [18] and Chaperon [11] for the compact case. Later, Viterbo [21] built up a global version in the non-compact case $\mathbb{R}^{n}$ using the reduction techniques of Amann, Conley and Zehnder [2, 12]. Viterbo's theorem states the existence of global generating function for Lagrangian submanifolds, geometric solutions of an evolution problem of Hamilton-Jacobi, starting from zero-section of $T^{*} \mathbb{R}^{n}$ with Hamiltonian function $H: T^{*} \mathbb{R}^{n} \rightarrow \mathbb{R}$ with second partial derivatives uniformly bounded. It is not yet available in literature a version for non-compact and non-parallelizable manifolds. The aim of Chaperon and Viterbo was mainly to construct a new global theory of weak solutions for Hamilton-Jacobi problem - the so-called min-max solutions based on the Lusternik-Schnirelman theory.

Here is a scheme for the proof of Viterbo's statement:

1. Viterbo's theorem describes Lagrangian submanifolds connected by a Hamiltonian isotopy to the zero-section of $T^{*} \mathbb{R}^{n}$. The curves in the Sobolev space $H^{1}\left([0, T], T^{*} \mathbb{R}^{n}\right)$, starting from zero section, are described by the couple $\left(q_{T}, \phi\right)$ where $q_{T}$ is the $q$-projection of the final point (at the time $t=T$ ) and $\phi \in L^{2}$ is the velocity of the curve.

2. The Action Functional is considered as a formal global generating function with infinite parameters (in $L^{2}$ ). In fact, the variation of the Action 
Functional (obtained with a variation of velocity) is zero exactly in correspondence to the solutions of the related Hamilton system.

3. Developing velocities in Fourier series and keeping the finite kernel (terms from $-N$ to $N$ ) and the infinite tail, one can observe that, for $N$ large enough, the finite kernel of velocity of a solution determines the full solution (by a fixed-point lemma). So, one may consider only a finite number of parameters, obtaining in this way a global generating function.

We notice that Viterbo considers curves starting from zero-section, i.e. with vanishing initial conjugate momentum, and constructs an object $\Lambda \subset T^{*} \mathbb{R}^{n}$. This fact simplifies the proof a lot. By minor changes, one could carry out the same construction by starting from any exact Lagrangian submanifold.

Now, in order to construct a generating function of a symplectic relation, that is, an object inside $T^{*} \mathbb{R}^{n} \times T^{*} \mathbb{R}^{n}$, we have to reformulate the framework drastically. Moreover, in the original theory all works for fixed initial vanishing momentum $p_{0}=0$ and fixed final configuration $q_{T}$. On the other side, since our aim here is to construct a two-points generating function with auxiliary parameters describing the above symplectic relation, we have to fix both initial and final configurations $q_{0}$ and $q_{T}$.

Curves will be identified by the the straight segment between $q_{0}$ and $q_{T}$ plus loops. The variations of a curve will be still given by loops. This approach via restoring loops can be found also in a recent paper [13].

It is worth noticing that, after the seminal paper [20] by Tulczyjew on the HamiltonJacobi theory, Hamilton Principal Functions with auxiliary parameters were considered for the first time in [5] and [7].

Under some suitable hypothesis, when the Lagrangian function $L$ is the (half of the) quadratic form valued on the 4-velocity related to a Riemannian or a SemiRiemannian metric, our construction leads to a generating function with auxiliary parameters which is exactly the announced globalization of the World Function of Synge, see Theorem 1 and Corollary 3. For the related Hamilton Principal Function, see Corollary 5.

\section{A Two-point Version of Viterbo's construction}

In this section $Q=\mathbb{R}^{n}$ and $H$ will denote a Hamiltonian function $H: T^{*} \mathbb{R}^{n} \rightarrow$ $\mathbb{R}$. Adopting standard notations, as in $[1,3,6]$, we denote by $\omega=\mathrm{d} p \wedge \mathrm{d} q=$ $\sum_{i=1}^{n} \mathrm{~d} p_{i} \wedge \mathrm{d} q^{i}$ the standard symplectic 2 -form on $T^{*} \mathbb{R}^{n}$. The Hamiltonian vector 
field $X_{H}$ is defined by $i_{X_{H}} \omega=-\mathrm{d} H$. Denoting curves by $\gamma=(q, p)$ and setting $J=\left(\begin{array}{cc}\mathbb{O} & \mathbb{I} \\ -\mathbb{I} & \mathbb{O}\end{array}\right)$, the related Hamilton equations $\dot{\gamma}=X_{H}(\gamma)=J \nabla H(\gamma)$ read

$$
\dot{q}=\frac{\partial H}{\partial p}(q, p), \quad \dot{p}=-\frac{\partial H}{\partial q}(q, p) .
$$

We denote by $\phi_{H}^{t}$ the flow of $X_{H}, \frac{\mathrm{d} \phi_{H}^{t}}{\mathrm{~d} t}=J \nabla H\left(\phi_{H}^{t}\right)$. We consider the standard projections

$$
T^{*} \mathbb{R}^{n} \stackrel{p r_{1}}{\longleftarrow} T^{*} \mathbb{R}^{n} \times T^{*} \mathbb{R}^{n} \stackrel{p r_{2}}{\longrightarrow} T^{*} \mathbb{R}^{n}
$$

and we carry out the symplectic structure $\bar{\omega}$ on $T^{*} \mathbb{R}^{n} \times T^{*} \mathbb{R}^{n} \cong T^{*}\left(\mathbb{R}^{n} \times \mathbb{R}^{n}\right)$ by the following twofold pull-back of the standard symplectic 2-form on $\mathbb{R}^{n}$

$$
\bar{\omega}:=p r_{2}^{*} \omega-p r_{1}^{*} \omega=\mathrm{d} p_{2} \wedge \mathrm{d} q_{2}-\mathrm{d} p_{1} \wedge \mathrm{d} q_{1} .
$$

We recall that the Characteristic Relation of the Hamiltonian system $H$ is the set $\mathcal{C}$ of points $\left(q_{1}, p_{1} ; q_{2}, p_{2}\right) \in T^{*} \mathbb{R}^{n} \times T^{*} \mathbb{R}^{n}$ such that, for some $T \in \mathbb{R}$, one has $\left(q_{2}, p_{2}\right)=\phi_{H}^{T}\left(q_{1}, p_{1}\right)$. It comes out that $\mathcal{C}$ is a Lagrangian submanifold of $\left(T^{*} \mathbb{R}^{n} \times T^{*} \mathbb{R}^{n}, \bar{\omega}\right)$, that is $\left.\bar{\omega}\right|_{\mathcal{C}}=0$ and $\operatorname{dim} \mathcal{C}=2 n=\frac{1}{2} \operatorname{dim}\left(T^{*} \mathbb{R}^{n} \times T^{*} \mathbb{R}^{n}\right)$.

Theorem 1. Let $H: T^{*} \mathbb{R}^{n} \rightarrow \mathbb{R}$ be a $C^{2}$ Hamiltonian function. Suppose the following condition is satisfied

$$
\sup _{(q, p) \in T^{*} \mathbb{R}^{n}}\left|\nabla^{2} H\right|=C<+\infty .
$$

Then the set $\Lambda \subset T^{*} \mathbb{R}^{n} \times T^{*} \mathbb{R}^{n}$ defined by

$$
\Lambda:=\left\{\left(q_{0}, p_{0}, q_{T}, p_{T}\right) \in T^{*} \mathbb{R}^{n} \times T^{*} \mathbb{R}^{n} ; \phi_{H}^{T}\left(q_{0}, p_{0}\right)=\left(q_{T}, p_{T}\right)\right\}
$$

admits a global generating function with finite auxiliary parameters

$$
S: \mathbb{R}^{n} \times \mathbb{R}^{n} \times \mathbb{R}^{k} \rightarrow \mathbb{R}, \quad\left(q_{0}, q_{T}, u\right) \mapsto S\left(q_{0}, q_{T}, u\right)
$$

such that:

$$
p_{0}=-\frac{\partial S}{\partial q_{0}}, \quad p_{T}=\frac{\partial S}{\partial q_{T}}, \quad 0=\frac{\partial S}{\partial u} .
$$

Remark 2. For general Hamiltonian systems, the set $\Lambda$ would not be the characteristic relation $\mathcal{C}$, since the final time $t=T$ is fixed. Anyway, this set $\Lambda$ is actually Lagrangian (maximal isotropic) with respect to the symplectic 2-form $\bar{\omega}$. In particular, for geodesic-like Hamiltonian $H=\frac{1}{2} g_{i j}^{-1}(q) p_{i} p_{j}, \Lambda$ coincides (by choosing $T=1$ ) precisely with the Characteristic Relation $\mathcal{C}$, see Corollary 3. 
The above requested boundness of the second derivatives of $H$ is a hard task to verify whenever the Hamiltonian function is not compactly supported, or definitively quadratic. In $\mathbb{R}^{2 n}$ this difficulty arises especially by concerning with geodesic problems (in the Riemannian or Semi-Riemannian case), for which

$$
H=\frac{1}{2} g^{-1}(q)(p, p), \quad \nabla^{2} H=\left(\begin{array}{cc}
\frac{1}{2} \frac{\partial^{2} g^{i j}(q)}{\partial q^{l} \partial q^{m}} p_{i} p_{j} & \frac{1}{2} \frac{\partial g^{i j}(q)}{\partial q^{l}} p_{i} \\
\frac{1}{2} \frac{\partial i^{i j}(q)}{\partial q^{l}} p_{i} & g^{i j}(q)
\end{array}\right)
$$

so that we cannot assume any 'a priori' boundness on $p$ 's, especially in the SemiRiemannian case (in the Riemannian one, which is a $p$-convex case, energy conservation does help us to it.)

In order to remove this difficulty and to avoid extreme sorts of pathology, we will restrict inside a fixed (large) compact sub-set $\mathcal{K} \subset \mathbb{R}^{n}$ together with the request of finite cardinality of the sets of geodesics linking the pairs $\left(q_{0}, q_{1}\right) \in$ $\mathcal{K} \times \mathcal{K}$. This property - see $(* *)$ below - seems to be reasonably enjoyed by Riemannian [Semi-Riemannian] metric tensors which are suitably asymptotic to Euclidean [Minkowskian] metrics. We do not delve further into this matter.

Warning: We are going to deal with

i) Riemannian $g=g_{R}, \operatorname{sgn}\left(g_{R}\right)=(1, \ldots, 1)$, and

ii) Semi-Riemannian $g=g_{S R}, \operatorname{sgn}\left(g_{S R}\right)=(-1,1, \ldots, 1)$ manifolds topologically equivalent to $\mathbb{R}^{n}$ with trivial atlas (one chart is sufficient).

Anyway, the Euclidean metric (and norm) many times used below could be replaced by minor changes

i) just by the Riemannian metric, and

ii) in the Semi-Riemannian case, by the following Riemannian metric $[15,17]$

$$
\hat{g}_{R}:=g_{S R}+2 u \otimes u
$$

where $u$ is a time-like vector field, $\left\langle g_{S R} u, u\right\rangle \equiv-1$, giving the timeorientation to $\left(\mathbb{R}^{n}, g_{S R}\right)$.

We are ready to state the following

Corollary 3. (World Function) Let $H: T^{*} \mathbb{R}^{n} \rightarrow \mathbb{R}, H=\frac{1}{2} g^{-1}(q)(p, p)$, be the $C^{2}$ Hamiltonian function of a Riemannian or Semi-Riemannian system. Consider the set of geodesics (for $T=1$ )

$$
\begin{aligned}
G\left(q_{0}, q_{1}\right) & =\left\{\gamma(\cdot)=(q(\cdot), p(\cdot)) \in H^{1}\left([0,1], \mathbb{R}^{2 n}\right) ;\right. \\
q(0) & \left.=q_{0}, \quad q(1)=q_{1}, \quad \dot{\gamma}=J \nabla H(\gamma)\right\} .
\end{aligned}
$$


For a fixed (arbitrarily large) compact $\mathcal{K}$ of $\mathbb{R}^{n}$, we suppose that

$$
\# G\left(q_{0}, q_{1}\right)<+\infty \quad \forall\left(q_{0}, q_{1}\right) \in \mathcal{K} \times \mathcal{K} .
$$

Then the above set $\Lambda$ in $(*)$, restricted to $T^{*} \mathcal{K} \times T^{*} \mathcal{K}$, is precisely the expected Lagrangian submanifold denoting the Characteristic Relation $\mathcal{C}$ of the Hamiltonian system $H$ and the related generating function $S$

$$
\mathcal{K} \times \mathcal{K} \times \mathbb{R}^{k} \ni\left(q_{0}, q_{1}, u\right) \mapsto S\left(q_{0}, q_{1}, u\right) \in \mathbb{R}
$$

represents the required generalized global World Function.

Remark 4. Alternatively, the hypothesis (**) may be replaced by the following one. There exists a compact set $\mathcal{G} \subset H^{1}$ such that

$$
G\left(q_{0}, q_{1}\right) \subset \mathcal{G} \quad \forall\left(q_{0}, q_{1}\right) \in \mathcal{K} \times \mathcal{K} .
$$

Indeed $H^{1} \hookrightarrow C^{0}$ (by Sobolev embedding) so $\mathcal{G}$ is bounded and we have an a priori bound on every point of each geodesic joining pairs of points of $\mathcal{K}$.

Finally, we have

Corollary 5. (Hamilton Principal Function) Let $S\left(q_{0}, q_{1}, u\right)$ be a generalized World Function related to the Hamiltonian $H: T^{*} \mathbb{R}^{n} \rightarrow \mathbb{R}, H=\frac{1}{2} g^{-1}(q)(p, p)$, for a Riemannian or Semi-Riemannian metric $g$ and $\left(q_{0}, q_{1}\right) \in \mathcal{K} \times \mathcal{K}$, where $\mathcal{K}$ is a compact set of $\mathbb{R}^{n}$. Then the function

$$
\mathcal{S}\left(q_{0}, q_{1}, u\right)=2 \sqrt{\left|e S\left(q_{0}, q_{1}, u\right)\right|}
$$

is a (generalized) global Hamilton Principal Function for the Hamilton-Jacobi equation $H=e$.

Remark 6. Given the above generalized World Function $S=S\left(q_{0}, q_{1}, u\right)$, if we are able to remove the auxiliary parameters by means of the stationarization

$$
\frac{\partial S}{\partial u}\left(q_{0}, q_{1}, u\right)=0
$$

we find the classical World Function again. In general, we cannot remove them and this fact is due to the lack of transversality of $\Lambda$ with respect the fibers of $\pi: T^{*}\left(\mathbb{R}^{n} \times \mathbb{R}^{n}\right) \rightarrow \mathbb{R}^{n} \times \mathbb{R}^{n}$, which tells us that there are more geodesics connecting $q_{0}$ and $q_{1}$. 
More precisely, it can happen that for the above relation $\partial S / \partial u=0$

1. there is no solution: we have no geodesics connecting $q_{0}$ and $q_{1}$

2. we find $h$ functionally independent solutions $u_{\alpha}=\tilde{u}_{\alpha}\left(q_{0}, q_{1}\right), \alpha \in\{1, \ldots, h\}$, i.e. we have $h$ geodesics connecting $q_{0}$ and $q_{1}$.

If the points $q_{0}$ and $q_{1}$ are close enough, we always have only one geodesic connecting them, $u=\tilde{u}\left(q_{0} q_{1}\right)$, so we restore the classical local World Function $\Omega\left(q_{0}, q_{1}\right):=S\left(q_{0}, q_{1}, \tilde{u}\left(q_{0}, q_{1}\right)\right)$, but the first conjugate point to $q_{0}$ along the prolungation of this geodesic (towards the future) signals the end of this condition of transversality.

Remark 7. Let us consider the Geometric Cauchy Problem in $\mathcal{K}$, for the HamiltonJacobi equation

$$
H \circ \mathrm{d} S=e
$$

for initial data $\sigma: \Sigma^{n-1} \rightarrow \mathbb{R}$ on the hypersurface $j: \Sigma^{n-1} \hookrightarrow \mathcal{K} \subset \mathbb{R}^{n}$ which can be represented by the following initial Lagrangian submanifold

$$
\Lambda_{0}=\left\{(q, p) ;\langle p, T j(\chi) v\rangle=\langle d \sigma, v\rangle, q=j(\chi), \quad \forall v \in T_{\chi} \Sigma^{n-1}, \quad \forall \chi \in \Sigma^{n-1}\right\} .
$$

It is solved - see [8] for details - by the Lagrangian manifold $\Lambda$ globally described by the following generating function $\hat{\mathcal{S}}$ with auxiliary parameters $(u, \chi)$

$$
\hat{\mathcal{S}}(q, u, \chi)=\mathcal{S}(\tilde{q}(\chi), q, u)+\sigma(\chi) .
$$

The global Hamilton Principal Function $\mathcal{S}\left(q_{0}, q_{1}, u\right)$ can be interpreted as a sort of Geometric Green Kernel for Hamilton-Jacobi problem.

\section{Proofs}

Proof of the Theorem 1. Let us consider $q_{0}, q_{T} \in \mathbb{R}^{n}$. We define the following set of curves $\left(H^{s} \equiv W^{s, 2}\right)$

$$
\Gamma=\left\{\gamma(\cdot)=(q(\cdot), p(\cdot)) \in H^{1}\left([0, T], \mathbb{R}^{2 n}\right) ; q(0)=q_{0}, q(T)=q_{T}\right\} .
$$

An element of $\Gamma$ is a curve in $T^{*} \mathbb{R}^{n}$ whose canonical projection on $\mathbb{R}^{n}$ is a curve connecting $q_{0}$ and $q_{T}$. A curve $\Gamma \ni \gamma:[0, T] \rightarrow \mathbb{R}^{2 n}$ solves the Hamiltonian system if the equation $\dot{\gamma}=J \nabla H(\gamma)$ is satisfied for almost every $t \in[0, T]$. Actually, by Sobolev embedding theorem, these curves are $C^{0}$. Given $\phi \in L^{2}\left([0, T], \mathbb{R}^{2 n}\right)$, 
for every fixed $N \in \mathbb{N}$, we consider two projection maps (which are Lipschitz with unitary norm)

$$
\begin{gathered}
\mathbb{P}_{N} \phi(s):=\sum_{|k| \leq N} \phi_{k} e^{\mathrm{i} \frac{2 \pi k}{T} s}, \quad \mathbb{Q}_{N} \phi(s):=\phi(s)-\mathbb{P}_{N} \phi(s) \\
L^{2}=\mathbb{P}_{N} L^{2} \oplus \mathbb{Q}_{N} L^{2}, \quad u \in \mathbb{P}_{N} L^{2}, \quad v \in \mathbb{Q}_{N} L^{2} .
\end{gathered}
$$

The subspaces $\mathbb{P}_{N} L^{2}$ and $\mathbb{Q}_{N} L^{2}$ are orthogonal with respect to the scalar product of $L^{2}$. Furthermore, we define the map

$$
h: \mathbb{R}^{n} \times \mathbb{R}^{n} \times L^{2} \longrightarrow \Gamma \quad\left(q_{0}, q_{T}, \phi(\cdot)\right) \longmapsto(q(t), p(t))=h\left(q_{0}, q_{T}, \phi(\cdot)\right)(t)
$$

where

$$
\left\{\begin{array}{l}
q(t):=q_{0}+\frac{t}{T}\left(q_{T}-q_{0}\right)+\int_{0}^{t}\left(\phi_{q}(s)-\frac{1}{T} \int_{0}^{T} \phi_{q}(\tau) \mathrm{d} \tau\right) \mathrm{d} s \\
p(t):=\frac{1}{T} \int^{T} \phi_{q}(\tau) \mathrm{d} \tau+\int_{0}^{t} \phi_{p}(s) \mathrm{d} s .
\end{array}\right.
$$

Essentially, $q(\cdot)$ is a variation of the straight line connecting $q_{0}$ and $q_{T}$. This is a bijective map, and indeed, by computing its inverse, we find

$$
\left\{\begin{aligned}
q_{0} & =q(0) \\
q_{T} & =q(T) \\
\phi_{q}(t)-\frac{1}{T} \int_{0}^{T} \phi_{q}(\tau) \mathrm{d} \tau & =\dot{q}(t)-\frac{1}{T}\left(q_{T}-q_{0}\right) \\
\phi_{p}(t) & =\dot{p}(t)
\end{aligned}\right.
$$

and the indeterminacy of the mean value of $\phi_{q}$, i.e. $\phi_{q}^{k=0}:=\frac{1}{T} \int_{0}^{T} \phi_{q}(\tau) \mathrm{d} \tau$, is fully removed

$$
p(0)=\phi_{q}^{k=0} .
$$

Now we consider the Hamilton-Helmholtz Action Functional

$$
\begin{gathered}
A: \mathbb{R}^{n} \times \mathbb{R}^{n} \times L^{2} \rightarrow \mathbb{R} \\
A\left[q_{0}, q_{T}, \phi\right]=\left.\int_{0}^{T}(p \cdot \dot{q}-H)\right|_{\gamma=h\left(q_{0}, q_{T}, \phi\right)} \mathrm{d} t
\end{gathered}
$$

Gateaux derivative $\frac{D A}{D \phi}\left[q_{0}, q_{T}, \phi\right] \delta \phi$ with $\delta \phi \in L^{2}\left(=T_{\phi} L^{2}\right)$ is defined by

$$
\frac{D A}{D \phi}\left[q_{0}, q_{T}, \phi\right] \delta \phi=\left.\frac{\mathrm{d} A}{\mathrm{~d} \lambda}\left(q_{0}, q_{T}, \phi+\lambda \delta \phi\right)\right|_{\lambda=0} .
$$


We have that

$$
\frac{D A}{D \phi}\left[q_{0}, q_{T}, \phi\right] \delta \phi=\int_{0}^{T}\left[\left(\dot{q}-\frac{\partial H}{\partial p}\right) \delta p-\left(\dot{p}+\frac{\partial H}{\partial q}\right) \delta q\right] \mathrm{d} s+\left.p \cdot \delta q\right|_{0} ^{T}
$$

where the variations $\delta q$ and $\delta p$ are deduced directly from the above definition of $h$, i.e.

$$
\begin{aligned}
& \delta q=\left.\frac{\mathrm{d}}{\mathrm{d} \lambda} q\left(\cdot, q_{0}, q_{T}, \phi+\lambda \delta \phi\right)\right|_{\lambda=0}=\int_{0}^{t}\left(\delta \phi_{q}(s)-\frac{1}{T} \int_{0}^{T} \delta \phi_{q}(\tau) \mathrm{d} \tau\right) \mathrm{d} s \\
& \delta p=\left.\frac{\mathrm{d}}{\mathrm{d} \lambda} p\left(\cdot, q_{0}, q_{T}, \phi+\lambda \delta \phi\right)\right|_{\lambda=0}=\delta \phi_{q}^{k=0}+\int_{0}^{t} \delta \phi_{p}(s) \mathrm{d} s .
\end{aligned}
$$

Notice that $\delta q(0)=\delta q(T)=0$. We obtain also

$$
\begin{aligned}
\frac{D A}{D \phi}\left[q_{0}, q_{T}, \phi\right] \delta \phi= & \int_{0}^{T}\left[\left(\dot{q}-\frac{\partial H}{\partial p}\right)\left(\delta \phi_{q}^{k=0}+\int_{0}^{t} \delta \phi_{p}(s) \mathrm{d} s\right)\right. \\
& \left.-\left(\dot{p}+\frac{\partial H}{\partial q}\right)\left(\int_{0}^{t}\left(\delta \phi_{q}(s)-\frac{1}{T} \int_{0}^{T} \delta \phi_{q}(\tau) \mathrm{d} \tau\right) \mathrm{d} s\right)\right] \mathrm{d} t .
\end{aligned}
$$

So the curve $\gamma(\cdot)=(q(\cdot), p(\cdot))$ solves the Hamiltonian equations if and only if $\frac{D A}{D \phi}\left[q_{0}, q_{T}, \phi\right] \delta \phi=0$ for every $\delta \phi \in L^{2}$. Along solutions we have

$$
\begin{aligned}
\frac{\partial A}{\partial q_{0}} & =\int_{0}^{T}\left[\left(\dot{q}-\frac{\partial H}{\partial p}\right) \frac{\partial p}{\partial q_{0}}-\left(\dot{p}+\frac{\partial H}{\partial q}\right) \frac{\partial q}{\partial q_{0}}\right] \mathrm{d} s+\left.p \cdot \frac{\partial q}{\partial q_{0}}\right|_{0} ^{T}=-p(0) \\
\frac{\partial A}{\partial q_{T}} & =\int_{0}^{T}\left[\left(\dot{q}-\frac{\partial H}{\partial p}\right) \frac{\partial p}{\partial q_{T}}-\left(\dot{p}+\frac{\partial H}{\partial q}\right) \frac{\partial q}{\partial q_{T}}\right] \mathrm{d} s+\left.p \cdot \frac{\partial q}{\partial q_{T}}\right|_{0} ^{T}=p(T) .
\end{aligned}
$$

This shows that the Action Functional may be regarded as a formal global generating function with infinite parameters (in $L^{2}$ ). Now we will prove the following 
Lemma 8. Under above hypothesis on $H$, for fixed $\left(q_{0}, q_{T}\right) \in \mathbb{R}^{n} \times \mathbb{R}^{n}$ and $u \in \mathbb{P}_{N} L^{2}$, we consider further the following map $\hat{h}_{N}$

$$
\hat{h}_{N}: \mathbb{Q}_{N} L^{2} \longrightarrow \mathbb{Q}_{N} L^{2} \quad v \longmapsto \hat{h}_{N}(v):=\mathbb{Q}_{N} J \nabla H\left(h_{N}\left(q_{0}, q_{T}, u+v\right)\right) .
$$

It is a contraction map for large enough $N$.

Proof. We recall that $\mathbb{Q}_{N} \operatorname{id}_{[0, T]}(t)=\sum_{|k|>N} \frac{\mathrm{i} T}{2 \pi k} e^{\mathrm{i} 2 \pi k t / T},\langle f(\cdot), g(\cdot)\rangle_{L^{2}([0, T])}:=$ $\int_{0}^{T} f(t) \bar{g}(t) \frac{\mathrm{d} t}{T}$, and $\|w(\cdot)\|_{L^{2}([0, T])}^{2}:=\langle w(\cdot), w(\cdot)\rangle_{L^{2}([0, T])}$. Writing briefly

$$
g(v):=h_{N}\left(q_{0}, q_{T}, u+v\right)
$$

and $v:=v_{2}-v_{1}$, for $v_{1}, v_{2} \in \mathbb{Q}_{N} L^{2}$, we get by direct computations

$$
\begin{aligned}
& \| g\left(v_{1}\right)-g\left(v_{2}\right)\left\|_{L^{2}} \leq\right\|\left(\int_{0}^{t}\left(v_{q 2}-v_{q_{1}}\right)(s) \mathrm{d} s, \int_{0}^{t}\left(v_{p^{2}}-v_{p_{1}}\right)(s) \mathrm{d} s\right) \|_{L^{2}} \\
&=\left\|\int_{0}^{t} v(s) \mathrm{d} s\right\|_{L^{2}} \leq\left\|\sum_{|k|>N} \frac{T}{\mathrm{i} 2 \pi k} v_{k} e^{\mathrm{i} 2 \pi k t / T}-\sum_{|k|>N} \frac{T}{\mathrm{i} 2 \pi k} v_{k}\right\|_{L^{2}} \\
& \leq \frac{T}{2 \pi N}\|v\|_{L^{2}}+\left\|\sum_{|k|>N} \frac{\mathrm{i} T}{2 \pi k} v_{k}\right\|_{L^{2}} \leq \frac{T}{2 \pi N}\|v\|_{L^{2}}+\left\|\mathbb{Q}_{N} \operatorname{Id}_{[0, T]}\right\|_{L^{2}}\|v\|_{L^{2}} \\
& \leq \frac{T}{2 \pi N}\|v\|_{L^{2}}+\frac{T}{2 \pi} \sqrt{\frac{2}{N}}\|v\|_{L^{2}}=\frac{T}{2 \pi N}(1+\sqrt{2 N})\|v\|_{L^{2}}, \\
&\left\|h\left(q_{0}, q_{T}, u+v_{2}\right)-h\left(q_{0}, q_{T}, u+v_{1}\right)\right\|_{L^{2}} \leq \frac{T}{2 \pi N}(1+\sqrt{2 N})\left\|v_{2}-v_{1}\right\|_{L^{2} .} .
\end{aligned}
$$

Finally, we can estimate:

$$
\begin{aligned}
& \left\|\mathbb{Q}_{N} J \nabla H\left(h\left(q_{0}, q_{T}, u+v_{2}\right)\right)-\mathbb{Q}_{N} J \nabla H\left(h\left(q_{0}, q_{T}, u+v_{1}\right)\right)\right\|_{L^{2}} \\
\leq & \sup _{\mathbb{R}^{2 n}}\left|\nabla^{2} H\right|\left\|g\left(v_{1}\right)-g\left(v_{2}\right)\right\|_{L^{2}} \leq \frac{C T}{2 \pi N}(1+\sqrt{2 N})\left\|v_{2}-v_{1}\right\|_{L^{2}} .
\end{aligned}
$$

For $N$ large enough, we have

$$
\frac{C T}{2 \pi N}(1+\sqrt{2 N})=: \alpha<1 .
$$

So this map is a contraction. The Banach-Caccioppoli Lemma ensures the existence of one and only one fixed point for this contraction. We will denote this fixed point by $f\left(q_{0}, q_{T}, u\right)$

$$
f\left(q_{0}, q_{T}, u\right)=\mathbb{Q}_{N} J \nabla H\left(h\left(q_{0}, q_{T}, u+f\left(q_{0}, q_{T}, u\right)\right)\right) .
$$


It can be proved that it depends smoothly on $q_{0}, q_{T}, u$. In fact, the fixed point function $f$ solves the equation for the unknown $v$

$$
\mathbf{G}\left(q_{0}, q_{T}, u, v\right):=\mathbb{Q}_{N} J \nabla H\left(h\left(q_{0}, q_{T}, u+v\right)\right)-v=0 .
$$

The implicit function theorem works, since

$$
\frac{\partial \mathbf{G}}{\partial v}\left(q_{0}, q_{T}, u, v\right)=\frac{\partial \mathbb{Q}_{N}}{\partial v} J \nabla H\left(h\left(q_{0}, q_{T}, u+v\right)\right)-\mathbb{I} .
$$

A bound for the derivatives - in the above r.h.s. is given by the contraction Lipschitz constant $\alpha$

$$
\left|\frac{\partial \mathbb{Q}_{N}}{\partial v} J \nabla H\left(h\left(q_{0}, q_{T}, u+v\right)\right)\right| \leq \alpha<1
$$

so that

$$
\left|\left[\frac{\partial \mathbf{G}}{\partial v}\left(q_{0}, q_{T}, u, v\right)\right]^{-1}\right| \leq \frac{1}{1-\alpha} .
$$

We gain that $f$ is differentiable with respect to $u, q_{0}, q_{T}$, e.g.

$$
\begin{aligned}
& \left|\frac{\partial f}{\partial u}\left(q_{0}, q_{T}, u, v\right)\right| \\
& =\left|-\left[\frac{\partial \mathbf{G}}{\partial v}\left(q_{0}, q_{T}, u, f\left(q_{0}, q_{T}, u\right)\right)\right]^{-1} \frac{\partial \mathbf{G}}{\partial u}\left(q_{0}, q_{T}, u, f\left(q_{0}, q_{T}, u\right)\right)\right| \\
& \leq \frac{\alpha}{1-\alpha}<\infty .
\end{aligned}
$$

Finally, it is crucial to observe that if we are able to solve the finite ${ }^{2}$ equation for

$$
\begin{aligned}
u=\left(\left(u_{q}^{k}\right)_{|k| \leq N},\left(u_{p}^{k}\right)_{|k| \leq N}\right) \in \mathbb{P}_{N} L^{2} \equiv \mathbb{R}^{k(n, N)} \\
\bar{u}=\mathbb{P}_{N} J \nabla H\left(h\left(q_{0}, q_{T}, u+f\left(q_{0}, q_{T}, u\right)\right)\right)
\end{aligned}
$$

where

$$
\bar{u}:=\left(u_{q}^{-N}, \ldots, u_{q}^{-1}, \frac{q_{T}-q_{0}}{T}, u_{q}^{1}, \ldots, u_{q}^{N},\left(u_{p}^{k}\right)_{|k| \leq N}\right)
$$

then, by adding term by term the above two relations $(\star)$ and $(\star \star)$, the curve $\gamma=h\left(q_{0}, q_{T}, u+f\left(q_{0}, q_{T}, u\right)\right)$ solves the Hamiltonian equations, its projection on $\mathbb{R}^{n}$ starts from $q_{0}$ and ends at $q_{T}$.

$$
{ }^{2} \operatorname{dim}\left(\mathbb{P}_{N} L^{2}\left([0, T], \mathbb{R}^{2 n}\right)\right)=2 n(N+1):=k(n, N)
$$


Remark 9. The above hypothesis of boundness of the second derivatives of $H$ could be weakened by an analogous Lipschitz boundness for the Hamiltonian vector field $X_{H}$, but in this case the differentiability of the fixed point $f$, inherited from the implicit function theorem, does not work anymore.

Lemma 10. The map

$$
\begin{aligned}
S: \mathbb{R}^{n} \times \mathbb{R}^{n} \times \mathbb{R}^{k(n, N)} & \rightarrow \mathbb{R} \\
\left(q_{0}, q_{T}, u\right) & \mapsto S\left(q_{0}, q_{T}, u\right):=A\left[q_{0}, q_{T}, u+f\left(q_{0}, q_{T}, u\right)\right]
\end{aligned}
$$

is a global generating function for $\Lambda$.

Proof. Put $\gamma=h\left(q_{0}, q_{T}, u+f\left(u, q_{0}, q_{T}\right)\right)=\left(q\left(\cdot, u, q_{0}, q_{T}\right), p\left(\cdot, u, q_{0}, q_{T}\right)\right)$, so that we have

$$
\begin{aligned}
\frac{\partial S}{\partial u} & =\int_{0}^{T}\left[\left(\dot{q}-\frac{\partial H}{\partial p}\right) \frac{\partial p}{\partial u}-\left(\dot{p}+\frac{\partial H}{\partial q}\right) \frac{\partial q}{\partial u}\right] \mathrm{d} s+\left.p \cdot \frac{\partial q}{\partial u}\right|_{0} ^{T} \\
& =\int_{0}^{T}(\dot{\gamma}-J \nabla H) \cdot J \nabla_{u} \gamma=\int_{0}^{T}\left(u-\mathbb{P}_{N} J \nabla H\right) \cdot J \nabla_{u} \gamma
\end{aligned}
$$

because $(\star)$ holds. The boundary term $\left.\frac{\partial q}{\partial u}\right|_{0} ^{T}$ vanishes, because

$$
q\left(0, u, q_{0}, q_{T}\right) \equiv q_{0}, \quad q\left(T, u, q_{0}, q_{T}\right) \equiv q_{T} .
$$

Now it is easy to see that if $\left(q_{0}, q_{T}, \phi\right) \in \mathbb{R}^{n} \times \mathbb{R}^{n} \times L^{2}$ satisfies

$$
p(0)=-\frac{\partial A}{\partial q_{0}}, \quad p(T)=\frac{\partial A}{\partial q_{T}}, \quad 0=\frac{D A}{D \phi}
$$

then $\left(q_{0}, q_{T}, u\right) \in \mathbb{R}^{n} \times \mathbb{R}^{n} \times \mathbb{R}^{k(n, N)}$ with $u=\mathbb{P}_{N} \phi$ satisfies

$$
p(0)=-\frac{\partial S}{\partial q_{0}}, \quad p(T)=\frac{\partial S}{\partial q_{T}}, \quad 0=\frac{\partial S}{\partial u} .
$$

On the other hand, if $\left(q_{0}, q_{T}, u\right) \in \mathbb{R}^{n} \times \mathbb{R}^{n} \times \mathbb{R}^{k(n, N)}$ satisfies the previous statement, then, setting $\phi=u+f\left(q_{0}, q_{T}, u\right)$, we have

$$
p(0)=-\frac{\partial A}{\partial q_{0}}, \quad p(T)=\frac{\partial A}{\partial q_{T}}, \quad 0=\frac{D A}{D \phi} .
$$


Note that the extremal momenta $p(0)$ and $p(T)$ are determined by the unique uniform continuous extension of $\gamma$.

Proof of the Corollary 3. We easily see that ${ }^{3}$

$$
\sup _{\left(q_{0}, q_{1}\right) \in \mathcal{K} \times \mathcal{K}} \sup _{\gamma \in G\left(q_{0}, q_{1}\right)} \sup _{t \in[0,1]}|\gamma(t)|=c<+\infty
$$

that is, $q(\cdot)$ and $p(\cdot)$ are bounded as well and the constant $c$ determines an upper bound for $\left|\nabla^{2} H\right|$ which is essential in the proof of the above Theorem 1. Indeed, arguing by contradiction, if $c=+\infty$, we could define a sequence $\left\{\left(q_{0}^{(m)}, q_{1}^{(m)}\right)\right\}_{m \in \mathbb{N}} \in \mathcal{K} \times \mathcal{K}$ such that

$$
\sup _{\gamma \in G\left(q_{0}^{(m)}, q_{1}^{(m)}\right)} \sup _{t \in[0,1]}|\gamma(t)|>m .
$$

By compactness, there exists a sub-sequence $\left\{\left(q_{0}^{\left(m_{l}\right)}, q_{1}^{\left(m_{l}\right)}\right)\right\}_{l \in \mathbb{N}}$ converging to $\left(\bar{q}_{0}, \bar{q}_{1}\right) \in \mathcal{K} \times \mathcal{K}$. Thus, between $\bar{q}_{0}$ and $\bar{q}_{1}$, there exists a finite number of geodesics $\bar{\gamma}_{\alpha} \in H^{1}, \alpha=1, \ldots, J<+\infty$, which by Sobolev theorem are continuous, hence

$$
\sup _{t \in[0,1] \alpha=1, \ldots, J}\left|\bar{\gamma}_{\alpha}(t)\right|=\max _{t \in[0,1] \alpha=1, \ldots, J}\left|\bar{\gamma}_{\alpha}(t)\right|=c<+\infty
$$

which is a contradiction.

If we suppose as in Remark 4 instead of finiteness, that all geodesics joining pairs of points of $\mathcal{K}$ are contained in a unique compact set $\mathcal{G} \subset H^{1}-$ see $(* *)^{\prime}$ and according to the Sobolev theorem we have

$$
\sup _{t \in[0,1] \gamma \in \mathcal{G}}|\bar{\gamma}(t)|=\sup _{\gamma \in \mathcal{G}}\|\gamma\|_{C^{0}} \leq c_{0} \sup _{\gamma \in \mathcal{G}}\|\gamma\|_{H^{1}} \leq c_{0} c_{1}<+\infty
$$

where $c_{0}, c_{1}>0$ are suitable constants.

As announced in the statement, we take definitively $T=1$. The generating function $S\left(q_{0}, q_{1}, u\right)$ so obtained is precisely the (generalized, with auxiliary parameters) World Function of the geodesic system. This is due to the invariance of the solutions of the geodesic equations with respect to the general affine transformations of the evolution parameter

$$
t=a \bar{t}+b, \text { for every fixed } a>0, b \in \mathbb{R} .
$$

\footnotetext{
${ }^{3}$ By completeness, if $G\left(q_{0}, q_{1}\right)=\emptyset$, we mean $\sup _{\gamma \in G\left(q_{0}, q_{1}\right)} \sup _{t \in[0,1]}|\gamma(t)|=0$.
} 
In such a case it is simple to see that $\left(H=\frac{1}{2} g_{i j}^{-1}(q) p_{i} p_{j}\right)$

$$
\begin{gathered}
\gamma(t)=(q(t), p(t)) \text { solves } \frac{\mathrm{d} \gamma}{\mathrm{d} t}(t)=J \nabla H(\gamma(t)) \text { if and only if } \\
\bar{\gamma}(\bar{t})=(\bar{q}(\bar{t}), \bar{p}(\bar{t})):=(q(t(\bar{t})), p(t(\bar{t})) a) \text { solves } \frac{\mathrm{d} \bar{\gamma}}{\mathrm{d} \bar{t}}(\bar{t})=J \nabla H(\bar{\gamma}(\bar{t})) .
\end{gathered}
$$

Finally, by recalling that $S$ is obtained by reduction of Hamilton-Helmholtz Action Functional $A$, from the invariance (cf. the original formula by Synge in the Introduction, and recalling also the Legendre transformation)

$\left.T \int_{t=0}^{t=T}(p \cdot \dot{q}-H)\right|_{\gamma} \mathrm{d} t=\left.\left(\bar{t}_{f i n}-\bar{t}_{i n}\right) \int_{\bar{t}_{i n}}^{\bar{t}_{f i n}}(p \cdot \dot{q}-H)\right|_{\bar{\gamma}} \mathrm{d} \bar{t}, \bar{t}_{i n}=-\frac{b}{a}, \bar{t}_{f i n}=\frac{T-b}{a}$

we have that (see Lemma 10)

$$
S\left(q_{0}, q_{1}, u\right)=\left.\int_{t=0}^{t=1}(p \cdot \dot{q}-H) \mathrm{d} t\right|_{\gamma=h\left(q_{0}, q_{1}, u+f\left(q_{0}, q_{1}, u\right)\right)}
$$

represents exactly the (generalized, with auxiliary parameters) Synge World Function.

Proof of the Corollary 5. From the very definition of $S\left(q_{0}, q_{1}, u\right)$, along the geodesics, it holds that

$$
S=\left.\int_{0}^{1}(p \cdot \dot{q}-H) \mathrm{d} t\right|_{\gamma=h\left(q_{0}, q_{1}, u+f\left(q_{0}, q_{1}, u\right)\right)}=\left.\int_{0}^{1} L \mathrm{~d} t\right|_{\gamma=h}=\left.\int_{0}^{1} H \mathrm{~d} t\right|_{\gamma=h}
$$

and therefore

$$
S\left(q_{0}, q_{1}, u\right)=H\left(q_{1}, \frac{\partial S}{\partial q_{1}}\left(q_{0}, q_{1}, u\right)\right), \quad \frac{\partial S}{\partial u}\left(q_{0}, q_{1}, u\right)=0 .
$$

In particular: $\operatorname{sgn}(H)=\operatorname{sgn}(S)$. For $\frac{\partial \mathcal{S}}{\partial u}\left(q_{0}, q_{1}, u\right)=0$, we compute

$$
H\left(q_{1}, \frac{\partial \mathcal{S}}{\partial q_{1}}\left(q_{0}, q_{1}, u\right)\right)=\frac{1}{2} g^{i j}\left(q_{1}\right) \frac{|e| \frac{\partial S}{\partial q_{1}^{i}} \frac{\partial S}{\partial q_{1}^{j}}}{|S|}=|e| \frac{H}{|H|}=|e| \operatorname{sgn} H
$$

so finally $H=e$. 


\section{Acknowledgments}

We would like to thank F. Giannoni, M. Spera and A. Turco for useful discussions and suggestions.

\section{References}

[1] Abraham R. and Marsden J., Foundations of Mechanics, Benjamin/Cummings Publishing Company, Reading, Mass., 1978.

[2] Amann H. and Zehnder E., Periodic Ssolutions of Asymptotically Linear Hamiltonian Systems, Manus. Math. 32 (1980) 149-189.

[3] Arnol'd V., Mathematical Methods of Classical Mechanics, Springer, Berlin, 1978.

[4] Bardi M. and Capuzzo-Dolcetta I., Optimal Control and Viscosity Solutions of Hamilton-Jacobi-Bellman Equations. Systems \& Control: Foundations \& Applications, Birkhäuser, Boston, MA, 1997.

[5] Benenti S., Symplectic Relations in Analytical Mechanics, In: Proceedings of the IUTAM-ISIMM Symposium on Modern Developments in Analytical Mechanics, Vol. I (Torino, 1982). Atti Accad. Sci. Torino Cl. Sci. Fis. Mat. Natur. 117 (1983), suppl. 1, 39-91.

[6] Benenti S., Hamiltonian Optics and Generating Families, Bibliopolis, Napoli, 2004.

[7] Benenti S. and Tulczyjew W., The Geometric Meaning and Globalization of the Hamilton-Jacobi Method, In: Differential Geometric Methods in Mathematical Physics (Proc. Conf., Aix-en-Provence/Salamanca, 1979), Lecture Notes in Math., Springer, Berlin, 836 (1980) pp. 9-21.

[8] Cardin F., On the Geometric Cauchy Problem for the Hamilton-Jacobi Equation, Il Nuovo Cimento B 104 (1989) 525-544.

[9] Cardin F., On Viscosity and the Geometric Solutions for the Hamilton-Jacobi Equations, Nonlinear Analysis, T.M.A. 20 (1993) 713-719.

[10] Cardin F., The Global Finite Structure of Generic Envelope Loci for Hamilton-Jacobi Equations, J.M.P. 43 (2002) 417-430.

[11] Chaperon M., Une idée du type géodésique brisées pour les systèmes hamiltoniens, C.R.A.S. (Paris) Série I, 312 (1984) 293-296.

[12] Conley C. and Zehnder E., Morse Type Index Theory for Flows and Periodic Solutions for Hamilton Equations, C.P.A.M. 37 (1984) 207-253. 
[13] Ekeland I., Ghoussoub N. and Tehrani H., Multiple Solutions for a Classical Problem in the Calculus of Variations, J. Diff. Eqs. 131 (1996) 229-243.

[14] de Felice F. and Clarke C., Relativity on Curved Manifolds, Cambridge Monographs on Mathematical Physics, Cambridge, 1990.

[15] Hawking S. and Ellis G., The Large Scale Structure of Space-Time, Cambridge University Press, Cambridge, 1973.

[16] Laudenbach F., Immersions lagrangiennes et fonctions génératrices., In: Aspects dynamiques et topologiques des groupes infinis de transformation de la mécanique (Lyon, 1986), Travaux en Cours, Hermann, Paris, 25 (1987) 79-85.

[17] Giannoni F., Masiello A. and Piccione P., A Morse Theory for Massive Particles and Photons in General Relativity, JGP 35 (2000) 1-34.

[18] Sikorav J., Problèmes d'intersections et de points fixes en géometrie hamiltonienne, Comm. Math. Helv. 62 (1987) 62-73.

[19] Synge J., Relativity: the General Theory, North Holland, Amsterdam, 1960.

[20] Tulczyjew W., Relations symplectiques et les équations d'Hamilton-Jacobi relativistes, C.R.A.S. (Paris) 281 A (1975) 545-547.

[21] Viterbo C., Recent Progress in Periodic Orbits of Autonomous Hamiltonian Systems and Applications to Symplectic Geometry, In: Nonlinear Functional Analysis (Newark, NJ, 1987), Lecture Notes in Pure and Appl. Math., Dekker, New York 121 (1990) pp. 227-250.

Dipartimento di Matematica

Pura ed Applicata

Università di Padova

via Belzoni 7

35131 Padova

ITALIA

E-mails: cardin@math.unipd.it amarigo@math.unipd.it 